\title{
As Kibs e a inovação tecnológica das firmas de serviços ${ }^{1}$
}

\author{
Luis Claudio Kubota ${ }^{2}$
}

\section{Resumo}

No âmbito da União Europeia e da OCDE, é crescente a importância atribuída aos intangíveis e aos serviços na chamada Economia Baseada no Conhecimento. Algumas firmas de serviços são classificadas como Knowledge Intensive Business Services (Kibs), ou seja, serviços empresariais intensivos em conhecimento. Segundo a literatura, as Kibs exercem um papel de facilitadoras da inovação, ao fornecerem a interface entre o conhecimento genérico disponível na economia e o conhecimento tácito localizado na firma. O estudo utilizou microdados da Pesquisa da Atividade Econômica Paulista (Paep) 2001 para investigar empiricamente se é possível afirmar que as Kibs contribuem para a inovação tecnológica de suas clientes do próprio setor de serviços. Os resultados indicam que os serviços para os quais essa afirmativa encontra respaldo foram: jurídicos, contábeis, de soluções de internet, de comunicação empresarial, de publicidade e propaganda e de gestão empresarial.

Palavras-chave: Kibs; Inovações tecnológicas; Indústria de serviços.

\section{Abstract}

\section{Kibs and technological innovation of service firms}

For the European Union and the OECD, a great importance is given to the intangibles and services in the so called Knowledge Based Economy. Some services firms are classified as Knowledge Intensive Business Firms (Kibs). According to the literature, Kibs act as facilitators of innovation, because they function as interfaces of the generic knowledge available in the economy, and the tacit knowledge of the firms. The study explored microdata from the Survey of the Economic Activity in São Paulo (Paep) 2001, to investigate empirically if it is correct to state that Kibs contribute to the technological innovation of their clients of the services sector. The results indicate that the services for which this statement is true are: juridical, accountancy, internet solutions, business communications, advertisement and business management.

Key words: Kibs; Technological innovation; Services firms.

JEL L84, O33.

\section{Introdução}

O setor de serviços historicamente foi marginalizado no âmbito dos estudos em economia, no Brasil e em outros países. Entretanto, alguns fatores contribuem para a mudança desse cenário. Em primeiro lugar, é crescente a importância do setor de serviços, tanto em países desenvolvidos quanto em desenvolvimento. Para a Organization for Economic Co-operation and Development - OECD (2005a), o incremento da produtividade e do emprego são

(1) Trabalho recebido em dezembro de 2006 e aprovado em maio de 2008.

(2) Instituto de Pesquisa Econômica Aplicada (Ipea), Brasília, DF. E-mail: luis.kubota@ ipea.gov.br. 
Luis Claudio Kubota

altamente dependentes do sucesso das empresas de serviços, que são importantes agentes do crescimento econômico recente de muitas economias dos países que fazem parte daquela organização.

No Brasil, o setor serviços foi o maior receptor dos ingressos brutos de Investimento Externo Direto (IED) até setembro de 2007, absorvendo 47,3\% do total direcionado ao país, US\$ 11,2 bilhões. Esse resultado refletiu as elevações registradas nos segmentos intermediação financeira, serviços prestados a empresas, comércio, construção, transportes, correios e telecomunicações, e atividades de informática. O IED destinado ao setor industrial totalizou US\$ 9,4 bilhões, 39,5\% do total, segundo dados da Secretaria de Comunicação Social da Presidência da República.

A literatura sobre inovação em serviços é bastante convergente em suas principais propostas. Os mais destacados autores defendem que o setor de serviços é inovativo, apesar de as inovações apresentarem, em geral, um caráter menos tecnológico comparando-se com o que se observa na indústria. Os estudiosos do setor defendem a importância das inovações organizacionais, e apontam para a deficiência dos instrumentos de pesquisa baseados em modelos voltados para a indústria em apreender as especificidades dos serviços. Ainda não existe um modelo conceitual que seja amplamente aceito na explicação do fenômeno. A literatura defende que o estudo sobre a inovação em serviços pode contribuir para o entendimento dos fenômenos inovativos na indústria, até pelo fato de esse setor empreender uma série de atividades de serviços.

Uma crescente corrente da literatura está interessada em investigar o papel das Kibs enquanto indutoras do processo de inovação em firmas de outros setores (Nählinder, 2002; Bilderbeek et al., 1998; Kox, 2002; Hertog, 2000 e Antonelli, 1998). Segundo esses autores, as Kibs exercem um papel de facilitadores do processo de inovação na economia, inclusive em outros setores que não o de serviços. Segundo Antonelli (1998), Katsoulacos e Tsounis (2000) e Tomlinson (2000), as Kibs - assim como os serviços de informação e comunicação contribuem para o incremento da produtividade das economias. ${ }^{3}$

De acordo com Lööf (2000), os modernos processos de inovação são caracterizados por uma crescente complexidade e interdependência entre os diferentes atores, que combinam diversos tipos de conhecimento. Vários problemas na busca de inovação podem ser superados por um processo no qual a firma realiza um escrutínio de suas próprias capacidades e fraquezas, e depois identifica potenciais parceiros - que incluem consumidores e fornecedores - numa

(3) O primeiro autor estudou as economias europeias; Katsoulacos e Tsounis, a economia grega, e Tomlinson fez uma comparação entre o caso britânico e o japonês. 
crescente importância das redes informais que são caracterizadas pela livre distribuição de conhecimento.

Por meio da Pesquisa da Atividade Econômica Paulista (Paep) 2001, da Fundação Sistema Estadual de Análise de Dados (Seade), o presente estudo busca investigar se existe efetivamente a contribuição das Kibs para o processo de inovação de suas clientes do próprio setor de serviços. Este artigo está organizado da seguinte forma: na Seção 1, é apresentado o referencial teórico sobre inovação em serviços e a contribuição das Kibs para o processo inovativo. Na Seção 2, é apresentada a metodologia. Na Seção 3, são apresentados os resultados. Na sequencia as conclusões e as referências bibliográficas.

\section{Referencial teórico}

O tema da inovação em serviços ganha crescente atenção na literatura internacional, especialmente no contexto dos países da União Europeia. Um exemplo desse esforço é o projeto Services In Innovation, Innovation in Services Services in European Innovation Systems (SI4S), patrocinado pela Comunidade Europeia. Outro exemplo é o projeto Enhancing the Performance of the Service Sector, da OECD.

Ao contrário do que ocorre no Brasil, as pesquisas nacionais de inovação tecnológica (Community Innovation Surveys, baseadas no Manual de Oslo da OECD) já incorporam há anos o setor de serviços, o que permite uma série de análises nacionais, bem como comparações internacionais. Vide, por exemplo: Tether et al. (2002); Hipp et al. (2000); Tether e Miles (2000); Sundbo e Gallouj (1998). Não obstante o criticismo com relação a deficiências dessas pesquisas de inovação em captar as particularidades do setor de serviços (Djellal; Gallouj, 2000; Nählinder, 2002; Miles, 2000; Bilderbeek et al., 1998), trata-se de uma enorme vantagem em relação ao caso brasileiro, onde se pode destacar a Pesquisa da Atividade Econômica Paulista (Paep) 2001, que analisa a inovação tecnológica das empresas de serviços que atuam no Estado de São Paulo. Somente muito recentemente, a Pesquisa de Inovação Tecnológica 2005 incluiu os seguintes setores de serviços: telecomunicações, informática e pesquisa e desenvolvimento.

Sundbo e Gallouj (1998) definem inovação como uma mudança nos negócios pela adição de um novo elemento, ou pela combinação de elementos velhos em um sentido schumpeteriano. A inovação deve ser um fenômeno de alguma dimensão para ser considerada como tal. Isso significa que a mudança deve ser reproduzível e realizada mais de uma vez.

Os autores definem quatro tipos de inovação em serviços: de produto, de processo, organizacional e de mercado. Como exemplo de inovação organizacional, pode-se citar a introdução de gerência da qualidade total. As 
inovações de processo são renovações de procedimentos para produzir e entregar o serviço, e podem ser subdivididas em duas categorias: no processo de produção ("back office") e no processo de entrega ("front office"). Inovações de mercado são novos comportamentos mercadológicos, como, por exemplo, encontrar um novo segmento ou ingressar em outra indústria. Os autores sugerem uma outra forma de inovação, chamada de ad hoc, ou seja, a construção socialmente interativa para um problema particular proposto pelo cliente. Ela não é diretamente reproduzível, mas sim indiretamente através de codificação e formalização da experiência e competência. Para Drejer (2004), a inovação ad hoc não pode ser considerada como tal, visto que isso equivaleria a dizer que aprendizado, desenvolvimento de competência e codificação de conhecimento correspondem a inovações.

Sundbo e Gallouj (1998) apresentam alguns resultados da análise das pesquisas nacionais de inovação em serviços realizadas no âmbito do projeto SI4S, que incluiu os seguintes países: Dinamarca, França, Alemanha, Holanda, Noruega, Suécia e Grã-Bretanha. As maiores firmas tendem a ser mais inovadoras. As firmas que se internacionalizam tendem a ser mais inovativas, em função da troca de ideias. Falta de mão de obra qualificada, de recursos financeiros e de habilidade organizacional são os principais gargalos para o processo inovativo. As inovações tendem a ser simples e incrementais, e são facilmente imitáveis por competidores ágeis. Esse caráter incremental, em oposição à inovação em etapas, mais característica da indústria, é desenvolvido por Tether (2004) e Gallouj e Weinstein (1997).

Pesquisa e desenvolvimento (P\&D) formal e - de modo mais amplo - o caráter tecnológico da inovação não são características tão importantes para o processo inovativo no setor de serviços, em relação à manufatura (Cainelli; Evangelista; Savona, 2004; Sundbo; Gallouj, 1998; Lopes; Dodinho, 2005; Miles, 2000; Tether, 2004 e Gallouj, 2002). Por outro lado, as inovações organizacionais são consideradas como muito importantes por um grande número de autores (Miles, 2000; Sundbo; Gallouj, 1998; Gallouj, 2002; Gallouj; Weinstein, 1997; Tether, 2004; Hipp et al., 2000; Ark; Broersma; Hertog, 2003; Hertog; Broersma; Ark, 2003 e Hertog, 2003). A importância da força de trabalho e dos clientes para a inovação também encontra uma ampla ressonância entre os autores (Sundbo; Gallouj, 1998; Bilderbeek et al., 1998; Gallouj; Weinstein, 1997 e Gallouj, 2002).

Para a OECD (2005a), as inovações em serviços têm as seguintes características: (i) dependem menos de investimentos em P\&D formal, e mais de aquisição de conhecimento através da compra de equipamentos, propriedade intelectual, assim como por meio de colaboração; (ii) o desenvolvimento de recursos humanos é particularmente importante para os serviços, e a falta de mão de obra especializada pode ser um gargalo para a inovação na maior parte dos 
países da OECD; (iii) empresas menores tendem a ser menos inovativas que as maiores, mas o empreendedorismo é um fator que favorece a inovação; (iv) proteção de propriedade intelectual é um tema que merece atenção, especialmente no que diz respeito a software e métodos de negócios.

De acordo com Miles (2001), a literatura sobre serviços vem crescendo de modo intenso desde os meados dos anos 1960. Na ocasião, a visão predominante encarava os serviços como atrasados do ponto de vista tecnológico. Segundo Miles (2000), os esforços da Comunidade Europeia em estudar o setor de serviços remontam ao início dos anos 1980, com o Forecasting and Assessment of Science and Technology Programme (Fast). Naquele contexto, Miles (2001) destaca dois pesquisadores britânicos: Jay Gershuny e Richard Barras.

Gershuny efetivamente aceitava a visão de que os serviços eram em grande maioria não inovadores. O pesquisador cunhou o termo "self-service economy", uma tendência dos consumidores de produzirem os seus próprios serviços. Apesar dessa visão negativa, Gershuny vislumbrava a possibilidade de a tecnologia de informação propiciar a melhoria nos custos e na qualidade dos serviços.

Barras (1986), por sua vez, focou nas inovações no setor de serviços baseadas em tecnologia da informação (TI). Ele introduziu a teoria do ciclo reverso do produto (reverse product cycle - RPC), elaborada a partir da observação dos serviços financeiros na Grã-Bretanha. O RPC admite que os serviços são inovativos, especialmente através da aplicação de tecnologias de TI.

Miles (2001) ressalta essa ênfase em tecnologia na literatura anglofônica, em contraste com os autores francofônicos (como Gallouj; Weinstein, 1997), especialmente da Universidade de Lille, cuja ênfase se dá no caráter interativo dos serviços. Alguns autores enquadram abordagens como a de Lille como de "demarcação", que defende que a inovação em serviços tem características próprias muito distintivas em relação à manufatura. A abordagem de "assimilação" considera a inovação em serviços como fundamentalmente similar à da indústria. E a abordagem da "síntese" defende que as inovações em serviços e manufatura não seguem trajetórias completamente distintas, e que estudos que ressaltam os resultados das pesquisas de inovação em serviços podem contribuir para o entendimento da inovação na indústria. A proposta da abordagem da síntese materializou-se na última versão do Manual de Oslo: “... the scope of what is considered an innovation has now been expanded to include two new types: marketing and organisational innovation" (OECD, 2005b).

A mais consistente crítica ao RPC partiu de Uchupalanan (2000), que desenvolveu - a partir de estudos de caso no mercado financeiro tailandês - o Dynamic Interdependence of Innovation and Competition (DIIC) framework. 
Segundo o pesquisador tailandês, o RPC apresenta as seguintes deficiências: considera uma única fonte de inovação (TI), apresenta uma dicotomia entre produto e processo, falta de definição precisa do que é um novo serviço, as firmas de serviços são recipientes passivos de inovação dos fornecedores de TI. O modelo ignora o escopo de melhora simultânea de eficiência, qualidade e introdução de novos serviços.

Soete e Miozzo (2001) caracterizaram as empresas de serviços em três categorias, com relação às suas características tecnológicas: (i) Setores dominados pelos fornecedores, tais como educação e serviços pessoais. As firmas desse setor dão apenas pequenas contribuições para seu processo tecnológico, e a maior parte das inovações é oriunda de fornecedores de equipamentos, materiais e informação. (ii) Setores de redes físicas intensivas em escala ou que fazem parte de redes de informação, tais como fornecimento de gás e bancos. As inovações tecnológicas normalmente se originam da indústria, mas a natureza dessas inovações é fortemente determinada pelo seu uso nas firmas de serviços. (iii) Setores fornecedores de tecnologias especializadas ou baseadas em ciência, tais como software e laboratórios. As principais fontes de tecnologia são pesquisa, desenvolvimento e atividades de software do próprio setor.

Segundo Hertog (2000), as Kibs formam uma categoria de atividades de serviços que são altamente inovativas, e que, além disso, contribuem como facilitadores de inovação em outros setores econômicos. Elas são caracterizadas por serem organizações privadas, especializadas em determinado expertise técnico, e que fornecem produtos e serviços baseados em conhecimento.

Para Antonelli (1998), o processo de mudança tecnológica resulta da manipulação de dois tipos de conhecimento: genérico (conhecimento tecnológico codificado com conteúdo científico) e tácito (processos de aprendizado baseados em experiências específicas do inovador). Transformar o conhecimento localizado na firma em inovação bem-sucedida depende da capacidade de apropriar os resultados da pesquisa e desenvolvimento e de aprendizado interno, mas também da absorção sistemática de conhecimento disponível no ambiente da empresa. Nesse contexto, a rede de relações da firma com outros agentes ganha importância, e as Kibs, ao fornecerem a interface entre o conhecimento genérico disponível no sistema e o conhecimento tácito localizado na firma, podem exercer papel de extrema relevância. A difusão de tecnologias de inovação e conhecimento só veio corroborar nesse processo.

A relação entre as Kibs e suas clientes é classificada como simbiótica por Bilderbeek et al. (1998). Os profissionais que atuam nas Kibs ganham com a interação com os clientes, tanto quanto os próprios clientes. A experiência ganha em determinado projeto pode ser usada para o desenvolvimento de novos 
produtos, serviços e abordagens, e valoriza os profissionais envolvidos no caso de novos projetos similares. A maior parte das Kibs, pela natureza de sua operação, interage com um variado número de clientes, e muitas tornam-se intermediários importantes para os sistemas de inovação.

Para Hertog (2000), as Kibs exercem três funções:

a) Facilitadoras. As Kibs exercem papel de facilitadoras se elas suportam o cliente em seu processo inovativo, no caso em que elas não tenham sido as geradoras da inovação e não tenham transferido essa inovação de outras firmas para o cliente;

b) Transportadoras. As Kibs exercem papel de transportadoras quando elas transferem inovações de uma firma ou indústria para o cliente, mesmo que elas não tenham sido as geradoras da inovação; e

c) Fontes. As Kibs funcionam como fonte da inovação quando elas exercem um papel fundamental na iniciação e desenvolvimento da inovação no cliente, normalmente em processo interativo.

Nählinder (2002) alerta para o fato de que existem diversas formas de se operacionalizar a classificação das Kibs. O uso de classificações industriais apresenta a vantagem de possibilitar comparações ao longo do tempo. No caso brasileiro, Freire (2006) considerou as firmas que atuam nas seguintes divisões da Classificação Nacional de Atividade Econômica (CNAE ${ }^{4}$ contempladas na Pesquisa Anual de Serviços (PAS), ${ }^{5}$ como Kibs:

- Atividades de informática (divisão 72 da CNAE), as quais incluem as classes: Consultoria em sistemas de informática (7210), Desenvolvimento de programas de informática (7220), Processamento de dados (7230), Atividades de bancos de dados (7240), Manutenção e reparação de máquinas de escritório e de informática (7250).

- Telecomunicações (classe 6420 da CNAE).

- Serviços técnicos às empresas, grupo composto por classes selecionadas da divisão 74 da CNAE ("serviços prestados principalmente às empresas"), a saber: Atividades jurídicas (7411), Contabilidade e auditoria (7412), Pesquisa de mercado e de opinião pública (7413), Gestão de participação acionária (7414), Assessoria em gestão empresarial (7416), Serviços de arquitetura e engenharia e

(4) Em 2006, o Instituto Brasileiro de Geografia e Estatística (IBGE) realizou uma alteração nas divisões da CNAE. Entretanto, no presente artigo trabalharemos com a classificação anterior, que é a CNAE vigente na Paep 2001.

(5) A divisão 73 da CNAE, composta por "Pesquisa e desenvolvimento das ciências físicas e naturais" e "Pesquisa e desenvolvimento das ciências sociais e humanas", faz parte das Kibs, porém ficou de fora do estudo de Freire (2006) por não ser contemplada pela PAS, do Instituto Brasileiro de Geografia e Estatística (IBGE). 
de assessoramento técnico especializado (7420), Ensaios de Materiais e de Produtos (7430), Publicidade (7440).

No estudo de Freire (2006), há algumas evidências de que as firmas industriais que contratam Kibs são mais inovativas que as que não contratam.

As abordagens mais modernas de políticas de inovação, desenvolvidas no âmbito da União Europeia (Lengrand et al., 2002), posteriormente acatadas pela OECD (OECD, 2005c), enfatizam as chamadas políticas de terceira geração, e revelam um papel importante para as Kibs e a inovação de serviços. A primeira geração de políticas via a inovação como um processo que se iniciava na pesquisa básica, passando pela $\mathrm{P} \& \mathrm{D}$ até chegar à introdução no mercado de um produto ou tecnologia resultante desse processo. A segunda geração é identificada com o conceito de Sistemas Nacionais de Inovação (SNI). Essa abordagem introduz a perspectiva de que a análise dos processos de produção, difusão e uso de ciência, tecnologia e inovação (C,T\&I) deve considerar a influência simultânea de aspectos organizacionais, institucionais e econômicos. A terceira geração prega uma ação mais integrada das políticas de inovação com outras políticas - como a ambiental, de educação e de saúde - o que resulta na difícil tarefa de alinhar as necessidades de diferentes ministérios.

No âmago da abordagem europeia, está o conceito de Economia Baseada em Conhecimento (EBC). A EBC pode ser vista como uma interpretação das recentes tendências socioeconômicas, a visão do que a Europa deveria tornar-se, ou seja, um objetivo político. Três características da EBC têm consideráveis impactos na natureza da inovação: i) a emergência dos serviços e intangíveis; ii) o rápido desenvolvimento das tecnologias de informação e comunicação e a Sociedade da Informação; e iii) os novos papéis do conhecimento, aprendizado organizacional e recursos humanos.

Para Lengrand et al. (2002), a inovação em uma EBC não é baseada apenas em pesquisa e desenvolvimento e tecnologia, mas também em habilidades gerenciais e de mercadologia, e conhecimento organizacional, social, econômico e administrativo. Como os tipos e fontes de conhecimentos requeridos para inovações de maior porte tornam-se mais diversos, há maior ênfase na colaboração e uma tendência às inovações serem produzidas por uma rede de atores, em vez de serem desenvolvidas por indivíduos ou organizações autônomas. Conforme pode ser observado dessa discussão europeia e da OECD, a natureza mais incremental e menos tecnológica da inovação de serviços, e a importância da interação - e, por conseguinte, das Kibs - para a inovação, estão perfeitamente alinhadas com os mais recentes desenvolvimentos de políticas para inovação nos países desenvolvidos. 


\section{Metodologia}

\subsection{As hipóteses}

O presente estudo tem por objetivo identificar se as firmas de serviços que contratam Kibs são mais inovativas que suas congêneres que não o fazem. A seguir, apresentamos a hipótese nula principal, operacionalizada a partir das hipóteses nulas auxiliares. As diferentes Kibs contempladas nessas hipóteses foram selecionadas a partir da disponibilidade de questão a seu respeito no questionário da Paep, e posteriormente foi feito cruzamento dos setores considerados por Freire (2006) e respectivas CNAEs, já discutidas anteriormente.

$\mathrm{H}_{0}$ - As firmas de serviços que contratam Kibs não são mais inovativas que as congêneres que as contratam.

$\mathrm{H}_{0,1}$ - As firmas de serviços que contratam Kibs de assessoria jurídica não são mais inovativas que as congêneres que as contratam.

$\mathrm{H}_{0,2}$ - As firmas de serviços que contratam Kibs de contabilidade não são mais inovativas que as congêneres que as contratam.

$\mathrm{H}_{0,3}$ - As firmas de serviços que contratam Kibs de desenvolvimento de software não são mais inovativas que as congêneres que as contratam.

$\mathrm{H}_{0,4}$ - As firmas de serviços que contratam Kibs de processamento de dados não são mais inovativas que as congêneres que as contratam.

$\mathrm{H}_{0,5}$ - As firmas de serviços que contratam Kibs de gerenciamento de sites não são mais inovativas que as congêneres que as contratam.

$\mathrm{H}_{0,6}$ - As firmas de serviços que contratam Kibs de soluções de internet não são mais inovativas que as congêneres que as contratam.

$\mathrm{H}_{0,7}$ - As firmas de serviços que contratam Kibs de manutenção de computadores não são mais inovativas que as congêneres que as contratam.

$\mathrm{H}_{0,8}$ - As firmas de serviços que contratam Kibs de comunicação empresarial não são mais inovativas que as congêneres que as contratam.

$\mathrm{H}_{0,9}$ - As firmas de serviços que contratam Kibs de telemarketing não são mais inovativas que as congêneres que as contratam.

$\mathrm{H}_{0,10}$ - As firmas de serviços que contratam Kibs de publicidade e propaganda não são mais inovativas que as congêneres que as contratam.

$\mathrm{H}_{0,11}$ - As firmas de serviços que contratam Kibs de assessoria em gestão não são mais inovativas que as congêneres que as contratam. 
Luis Claudio Kubota

\subsection{A base de dados da Paep 2001}

No presente estudo, utilizou-se a Pesquisa da Atividade Econômica Paulista (Paep), realizada pela Fundação Seade em 2001, que abrange um amplo levantamento sobre as atividades econômicas das empresas do Estado de São Paulo, o mais rico e populoso do Brasil (mais de 40 milhões de habitantes), onde se concentra o maior número de empresas industriais e de serviços, incluindo o maior centro financeiro da América Latina. A Paep abrange os setores de manufatura, construção civil, serviços, comércio e bancos. O questionário completo, que abrange a questão da inovação tecnológica (conforme o Manual de Oslo), foi aplicado a empresas de cinco ou mais pessoas ocupadas, no caso da manufatura, e 20 ou mais pessoas ocupadas, no caso dos serviços. A amostra da manufatura representa uma população de 41 mil empresas, e a de serviços, de 21 mil empresas. A Paep considera também empresas com sede em outros estados da federação, desde que tenham ao menos uma unidade local produtiva em São Paulo, com mais de 30 pessoas ocupadas. Essas empresas fazem parte do estrato certo, assim como as firmas com sede no Estado de São Paulo com 100 ou mais pessoas ocupadas; as demais fazem parte do estrato amostral.

A classificação das empresas de serviços na Paep é mais abrangente que a da Pesquisa Anual de Serviços (PAS) do IBGE (2004), e inclui os setores de distribuição de energia elétrica, gás e água, educação, saúde, pesquisa e desenvolvimento e atividades associativas. A PAS, por sua vez, engloba os serviços auxiliares financeiros. $\mathrm{O}$ grande mérito da Paep é ser a primeira pesquisa de grande amostragem que contemplou a inovação no setor de serviços no Brasil. Somente muito recentemente, a Pintec 2005 do IBGE passou a incorporar alguns setores de serviços: telecomunicações, pesquisa e desenvolvimento e informática.

A maior parte das empresas pesquisadas no presente estudo - que preencheram o questionário completo da Paep - são de pequeno porte, e estão classificadas como segue, conforme a faixa de pessoal ocupado em 31/12/2001: até 29 pessoas ocupadas: 10.253 ; de 30 a 99 pessoas ocupadas: 8.080; de 100 a 499 pessoas ocupadas: 2.136; 500 pessoas ou mais: 794; total: 21.263. A receita líquida dessas empresas foi de $\mathrm{R} \$ 187,8$ bilhões em 2001, e o número de pessoas ocupadas em 31 de dezembro de 2001 foi de 2.604.136. A receita líquida obtida no Estado de São Paulo foi de R\$ 121 bilhões, e o pessoal ocupado no estado foi de 2.131.136.

A receita bruta obtida por todas as 361.078 empresas de serviços contempladas na Paep 2001 foi de $\mathrm{R} \$ 276,4$ bilhões, sendo que o valor gerado pelas firmas com sede no Estado de São Paulo foi de R $\$ 247,9$ bilhões, o que corresponde a $23,3 \%$ e 20,9\% do PIB de 2001, respectivamente. As empresas com sede em SP obtêm R \$ 87,2 bilhões, ou 35,2\% de suas receitas em outros estados. 
O total de pessoas ocupadas é de 3.790 .145 pessoas. As firmas com sede em SP empregam 3.641.993 pessoas, sendo 368.462, ou 10,1\%, fora do Estado.

Não obstante o fato de a literatura de inovação em serviços preconizar a importância das inovações organizacionais e não tecnológicas, o presente estudo concentrar-se-á nas inovações tecnológicas de produtos ou serviços, tendo em vista que esse é o foco da Paep 2001: produtos ou serviços que introduzam novas tecnologias. A Paep não considera inovações gerenciais ou organizacionais, bem como mudanças superficiais na prestação de serviços e no conceito de produtos ou serviços já existentes. O bloco 2 do questionário completo (Técnico-Produtivo), disponível no sítio da Seade, apresenta uma série de exemplos do que se considera inovação tecnológica na Paep. É possível observar que a complexidade do que pode ser considerado como inovação tecnológica é bastante variada, podendo representar desde a criação de peças de comunicação com a tecnologia Flash até sistemas de reconhecimento de fala, ou vacinas criadas a partir do Projeto Genoma. Entretanto, é importante lembrar que a pesquisa refere-se ao período de 1999 a 2001, quando muitas das tecnologias que hoje são triviais ainda eram pouco difundidas. Os resultados da pesquisa foram obtidos por meio de consulta aos microdados da Paep, na sala de acesso da Fundação Seade, e ao sítio da organização na internet. Consideraram-se no trabalho as respostas válidas das firmas com 20 ou mais pessoas ocupadas.

As atividades que compõem o setor de serviços são muito variadas, incorporando desde firmas que atuam em alojamento e alimentação, até empresas de grande porte dos setores de transporte e telefonia. O grau de inovação tecnológica para o mercado também é variado, sendo mais representativo nos setores de informática (30\%), pesquisa e desenvolvimento (22\%) e serviços relacionados com silvicultura e exploração florestal (12\%).

Para uma visão mais agregada, utilizou-se a classificação disponível na base de dados da Paep, que divide as firmas nos seguintes setores: telecomunicações (telecom), informática e conexas, pesquisa e desenvolvimento $(\mathrm{P} \& \mathrm{D})$, serviços técnicos prestados às empresas, serviços audiovisuais e outros serviços.

Na Tabela 1, pode-se observar que o fenômeno da inovação tecnológica é significativo para todos os setores de serviços. Entretanto, considerando-se apenas a inovação tecnológica para o mercado, destacam-se claramente os setores de telecomunicações, informática e pesquisa e desenvolvimento, sendo pouco expressiva nos demais setores. 
Luis Claudio Kubota

Tabela 1

Número e percentual de firmas de serviços inovadoras e inovadoras para o mercado por setor, entre 1999 e 2001

\begin{tabular}{l|r|r|r|r|r|r|r|r}
\hline & \multicolumn{4}{|c|}{ Inovadoras } & \multicolumn{3}{c}{ Inovadoras para o mercado } \\
\hline Setor & Não & $\%$ & Sim & $\%$ & Não & $\%$ & Sim & $\%$ \\
\hline Telecomunicações & 93 & $75 \%$ & 31 & $25 \%$ & 105 & $85 \%$ & 19 & $15 \%$ \\
\hline Informática & 614 & $64 \%$ & 352 & $36 \%$ & 677 & $70 \%$ & 287 & $30 \%$ \\
\hline P\&D & 31 & $76 \%$ & 10 & $24 \%$ & 32 & $78 \%$ & 9 & $22 \%$ \\
\hline $\begin{array}{l}\text { Serviços técnicos } \\
\text { prestados às empresas }\end{array}$ & 1.514 & $84 \%$ & 291 & $16 \%$ & 1.720 & $95 \%$ & 86 & $5 \%$ \\
\hline \begin{tabular}{l} 
Audiovisual \\
\hline
\end{tabular} & 435 & $84 \%$ & 80 & $16 \%$ & 503 & $98 \%$ & 11 & $2 \%$ \\
\hline \begin{tabular}{l} 
Outros serviços \\
\hline
\end{tabular} & 15.978 & $92 \%$ & 1.338 & $8 \%$ & 16.764 & $97 \%$ & 518 & $3 \%$ \\
\hline $\begin{array}{l}\text { Fonte: Paep/Seade (2001). Elaboração do autor a partir da transformação dos dados obtidos na } \\
\text { fonte. }\end{array}$
\end{tabular}

Na Tabela 2, pode-se observar que nos setores de informática, serviços técnicos prestados às empresas e outros serviços existe um percentual elevado de firmas que são as principais desenvolvedoras das inovações. Esse percentual é inferior a $50 \%$ nos setores de $\mathrm{P} \& \mathrm{D}$, telecomunicações e audiovisual. Utilizando a caracterização de Soete e Miozzo (2001), apenas o setor de audiovisual poderia ser inequivocamente considerado dominado pelos fornecedores, ou seja, cuja inovação é oriunda principalmente de provedores de equipamentos, materiais e informação.

Tabela 2

Número e \% de firmas de serviços inovadoras que foram as principais desenvolvedoras da inovação entre 1999 e 2001

\begin{tabular}{l|c|c|c|c}
\hline Setor & Não & $(\%)$ & Sim & $(\%)$ \\
\hline Telecomunicações & 19 & $61 \%$ & 12 & $39 \%$ \\
\hline Informática & 112 & $32 \%$ & 240 & $68 \%$ \\
\hline P\&D & 6 & $55 \%$ & 5 & $45 \%$ \\
\hline Serviços técnicos prestados às empresas & 121 & $42 \%$ & 170 & $58 \%$ \\
\hline Audiovisual & 71 & $89 \%$ & 9 & $11 \%$ \\
\hline Outros serviços & 596 & $46 \%$ & 707 & $54 \%$ \\
\hline
\end{tabular}

Fonte: Paep/Seade (2)001. Elaboração do autor a partir da transformação dos dados obtidos na fonte.

Observando-se a Tabela 3, pode-se verificar que, no setor de telecomunicações, apenas as licenças e patentes e empresas de consultoria têm baixa importância para menos de $50 \%$ das firmas inovadoras. Merecem destaque os departamentos da empresa, os fornecedores e os concorrentes como fonte de informação. 
Tabela 3

Número e percentual de firmas de serviços inovadoras que consideraram as fontes de informação para a inovação como importantes ou muito importantes ${ }^{6}$ entre 1999 e 2001

\begin{tabular}{|c|c|c|c|c|c|c|c|c|c|c|c|c|}
\hline \multirow{2}{*}{$\begin{array}{l}\text { Setor } \\
\text { Fonte informação }\end{array}$} & \multicolumn{2}{|c|}{ Telecom } & \multicolumn{2}{|c|}{ Informática } & \multicolumn{2}{|c|}{ P\&D } & \multicolumn{2}{|c|}{$\begin{array}{c}\text { Servs. } \\
\text { Empresariais }\end{array}$} & \multicolumn{2}{|c|}{ Audiovisual } & \multicolumn{2}{|c|}{ Outros } \\
\hline & $\begin{array}{c}\mathrm{N}^{\mathrm{o}} \\
\text { firmas }\end{array}$ & $(\%)$ & $\begin{array}{c}\mathrm{N}^{\circ} \\
\text { firmas }\end{array}$ & $(\%)$ & $\begin{array}{c}\mathrm{N}^{\circ} \\
\text { firmas }\end{array}$ & $(\%)$ & $\begin{array}{c}\mathrm{N}^{\mathrm{o}} \\
\text { firmas }\end{array}$ & $(\%)$ & $\begin{array}{c}\mathrm{N}^{\circ} \\
\text { firmas }\end{array}$ & $(\%)$ & $\begin{array}{c}\mathrm{N}^{\mathrm{o}} \\
\text { firmas }\end{array}$ & $(\%)$ \\
\hline Deptos. empresa & 27 & $90 \%$ & 319 & $91 \%$ & $*$ & $*$ & 177 & $64 \%$ & 52 & $65 \%$ & 1.015 & $76 \%$ \\
\hline Empresas grupo & 13 & $43 \%$ & 170 & $48 \%$ & 3 & $30 \%$ & 34 & $12 \%$ & 44 & $56 \%$ & 371 & $28 \%$ \\
\hline Fornecedores & 21 & $70 \%$ & 299 & $85 \%$ & $*$ & $*$ & 47 & $17 \%$ & 52 & $65 \%$ & 763 & $57 \%$ \\
\hline Clientes & 17 & $55 \%$ & 244 & $70 \%$ & 10 & $100 \%$ & 82 & $29 \%$ & 45 & $56 \%$ & 818 & $61 \%$ \\
\hline Concorrentes & 21 & $68 \%$ & 89 & $25 \%$ & $*$ & $*$ & 72 & $26 \%$ & 47 & $59 \%$ & 564 & $42 \%$ \\
\hline Consultoria & 10 & $32 \%$ & 80 & $23 \%$ & 4 & $40 \%$ & 45 & $16 \%$ & 48 & $60 \%$ & 388 & $29 \%$ \\
\hline Universidades & $*$ & $*$ & 119 & $34 \%$ & 7 & $64 \%$ & 29 & $10 \%$ & $*$ & $*$ & 296 & $22 \%$ \\
\hline Institutos pesquisa & $*$ & $*$ & 21 & $6 \%$ & 3 & $30 \%$ & 10 & $4 \%$ & 3 & $4 \%$ & 294 & $22 \%$ \\
\hline Licenças e patentes & 5 & $16 \%$ & 68 & $19 \%$ & $*$ & $*$ & 35 & $13 \%$ & 43 & $54 \%$ & 360 & $27 \%$ \\
\hline Conferências & 20 & $65 \%$ & 199 & $57 \%$ & $*$ & $*$ & 63 & $23 \%$ & 48 & $60 \%$ & 732 & $55 \%$ \\
\hline Feiras & 19 & $61 \%$ & 126 & $36 \%$ & 6 & $60 \%$ & 52 & $19 \%$ & 48 & $60 \%$ & 712 & $53 \%$ \\
\hline
\end{tabular}

Nota * Não divulgado em função de confidencialidade.

Fonte: Paep/SEADE 2001. Elaboração do autor a partir da transformação dos dados obtidos na fonte.

Com relação ao setor de informática, merecem destaque as mesmas fontes de informação do setor de telecomunicações. As conferências são importantes para $57 \%$ das empresas e todas as demais fontes ainda não citadas são importantes para menos de metade das empresas pesquisadas.

$\mathrm{O}$ setor de $\mathrm{P} \& \mathrm{D}$ tem várias fontes de informação que não podem ser divulgadas em função de confidencialidade. Entre as que constam da tabela, merece destaque a importância dos clientes como fonte de informação para a totalidade das firmas, bem como as universidades e feiras.

O setor de serviços técnicos prestados às empresas tem um comportamento bem distinto dos anteriores. Apenas os departamentos da empresa são (muito) importantes como fonte de informação para mais da metade das firmas pesquisadas.

No setor de audiovisual, o percentual de firmas que considera as fontes de informação como importantes varia entre $56 \%$ e $65 \%$, à exceção das universidades (não informado devido ao sigilo) e os institutos de pesquisa.

(6) Esta variável foi construída a partir das questões EA045 a EA057 do questionário. 
Luis Claudio Kubota

Nos Outros serviços, as seguintes fontes de informação foram consideradas (muito) importantes para mais de metade das firmas: departamentos da empresa, clientes, fornecedores, conferências e feiras.

O presente estudo procurará explorar a contribuição de alguns Fornecedores e Consultorias, além de outras Kibs, para o processo de inovação das firmas de serviços.

\section{Resultados}

Para se verificar a importância das Kibs e o caráter interativo do processo de inovação em serviços, desenvolveu-se o seguinte modelo logit de inputs para inovação:

$\mathrm{Li}=\operatorname{Ln}\{\mathrm{Pi}(\mathrm{Y}=1) /(1-\mathrm{Pi}(\mathrm{Y}=1)\}=\alpha+\beta 1 \mathrm{JUR}+\beta 2 \mathrm{CONT}+\beta 3 \mathrm{SOFT}+$ $\beta 4 \mathrm{DADO}+\beta 5 \quad \mathrm{SITE}+\beta 6 \mathrm{NET}+\beta 7 \quad \mathrm{COMP}+\beta 8 \mathrm{COM}+\beta 9 \mathrm{TMKT}+\beta 10$ $\mathrm{PROP}+\beta 11 \mathrm{GEST}+\beta 12 \mathrm{TESTi}+\beta 13 \mathrm{Ln}(\mathrm{PO}) \mathrm{i}+\beta 14 \mathrm{SETORi}+\mu \mathrm{i} \quad[1]$

- $\mathrm{Pi}(\mathrm{Y}=1)$ - probabilidade de a i-ésima observação ser classificada como inovadora. $^{7}$

- JUR - dummy que indica se a firma contratou - integral ou parcialmente serviços de assessoria jurídica prestados por terceiros (questão EW001, CNAE 7411). ${ }^{8}$

- CONT - dummy que indica se a firma contratou - integral ou parcialmente - serviços de contabilidade prestados por terceiros (questão EW003, CNAE 7416). ${ }^{8}$

- SOFT - dummy que indica se a firma contratou - integral ou parcialmente - serviços de desenvolvimento de sofware prestados por terceiros (questão EW018, CNAE 7220). ${ }^{8}$

-DADO - dummy que indica se a firma contratou - integral ou parcialmente - serviços de processamento de dados prestados por terceiros (questão EW019, CNAE 7240). ${ }^{8}$

- SITE - dummy que indica se a firma contratou - integral ou parcialmente - serviços de gerenciamento de sites prestados por terceiros (questão EW022, CNAE 7210). ${ }^{8}$

(7) A variável dependente foi construída a partir da questão EA003 do questionário: “A empresa introduziu no mercado algum serviço, produto e/ou processo tecnologicamente novo ou significativamente aperfeiçoado entre 1999 e 2001 ?’. Os valores missing não foram considerados.

(8) Variável considerada como "contrata de terceiros" para as firmas que responderam que contratam integral ou parcialmente de terceiros o referido serviço. Os valores missing foram inputados como "não". Codificada como $0=$ não e $1=$ sim. 
- NET - dummy que indica se a firma contratou - integral ou parcialmente serviços de solução de internet prestados por terceiros (questão EW023, CNAE 7210). ${ }^{8}$

- COMP - dummy que indica se a firma contratou - integral ou parcialmente - serviços de manutenção e conserto de computadores prestados por terceiros (questão EW025, CNAE 7250). ${ }^{8}$

- $\mathrm{COM}$ - dummy que indica se a firma contratou - integral ou parcialmente - serviços de comunicação empresarial prestados por terceiros (questão EW037, CNAE 7440). ${ }^{8}$

- TMKT - dummy que indica se a firma contratou - integral ou parcialmente - serviços de telemarketing prestados por terceiros (questão EW042, CNAE 7440). ${ }^{8}$

- PROP - dummy que indica se a firma contratou - integral ou parcialmente - serviços de publicidade e propaganda prestados por terceiros (questão EW044, CNAE 7440). ${ }^{8}$

- GEST - dummy que indica se a firma contratou - integral ou parcialmente - serviços de assessoria em gestão empresarial prestados por terceiros (questão EW045, CNAE 7416). ${ }^{8}$

- TEST - tempo de estudo médio da mão de obra (em anos), uma proxy para a qualificação da mão de obra. ${ }^{9}$ Espera-se que firmas com trabalhadores mais qualificados tenham maior propensão a inovar.

- Ln (PO) - Ln do pessoal ocupado, considerando não só o pessoal assalariado (questão EH025 do questionário), mas também os autônomos (EH037), terceiros alocados na empresa (EH038) e estatutários (EH039).

- SETOR - dummy que identifica o setor de atuação da firma, conforme classificação utilizada nas Tabelas 1,2 e $3 .{ }^{10} \mathrm{~A}$ introdução dessa variável tem a função de controlar as heterogeneidades setoriais que possam influenciar a inovação da firma. O setor de referência é o de "outros serviços".

- $\mu \mathrm{i}$ - é o símbolo dos resíduos da regressão.

A tabela a seguir apresenta os resultados do modelo:

(9) Esta variável foi construída a partir de cruzamento com informações da Relação Anual de Informações Sociais (Rais), do Ministério do Trabalho e Emprego, e da Pesquisa Anual de Amostra por Domicílios (PNAD) do IBGE.

(10) Inicialmente tentou-se utilizar uma abertura de divisão de atividade da CNAE a dois dígitos, entretanto, os modelos não convergiram. 
Luis Claudio Kubota

Tabela 4

Determinantes da probabilidade de a firma de serviços inovar

\begin{tabular}{|c|c|c|c|c|}
\hline Variáveis & Coeficiente & Erro- padrão & Wald & p-valor \\
\hline Constante & $-4,945$ & 0,275 & 322,688 & $<0,001$ \\
\hline $\mathrm{JUR}^{\mathrm{a}}$ & $-0,870$ & 0,092 & 88,814 & $<0,001$ \\
\hline $\mathrm{CONT}^{\mathrm{a}}$ & $-0,111$ & 0,069 & 2,588 & 0,108 \\
\hline SOFT $^{\mathrm{a}}$ & 0,010 & 0,075 & 0,019 & 0,890 \\
\hline $\mathrm{DADO}^{\mathrm{a}}$ & 0,327 & 0,083 & 15,620 & $<0,001$ \\
\hline SITE $^{\mathrm{a}}$ & $-0,124$ & 0,080 & 2,421 & 0,120 \\
\hline $\mathrm{NET}^{\mathrm{a}}$ & $-0,403$ & 0,078 & 26,526 & $<0,001$ \\
\hline $\mathrm{COMP}^{\mathrm{a}}$ & 0,419 & 0,079 & 28,080 & $<0,001$ \\
\hline $\mathrm{COM}^{\mathrm{a}}$ & $-0,154$ & 0,087 & 3,144 & 0,076 \\
\hline TMKT $^{\mathrm{a}}$ & $-0,141$ & 0,120 & 1,367 & 0,242 \\
\hline $\mathrm{PROP}^{\mathrm{a}}$ & $-0,310$ & 0,064 & 23,515 & $<0,001$ \\
\hline GEST $^{\mathrm{a}}$ & $-0,617$ & 0,067 & 87,737 & $<0,001$ \\
\hline TEST & 0,904 & 0,086 & 109,813 & $<0,001$ \\
\hline $\mathrm{Ln}(\mathrm{PO})$ & 0,356 & 0,027 & 168,957 & $<0,001$ \\
\hline SETOR & & & 363,939 & $<0,001$ \\
\hline Telecomunicações & 0,406 & 0,292 & 1,926 & $<0,001$ \\
\hline Informática & 1,796 & 0,097 & 345,172 & 0,020 \\
\hline $\mathrm{P} \& \mathrm{D}$ & 0,948 & 0,406 & 5,450 & $<0,001$ \\
\hline Serviços Técnicos & 0,657 & 0,098 & 44,782 & $<0,001$ \\
\hline Audiovisual & 0,897 & 0,169 & 28,238 & $<0,001$ \\
\hline \multicolumn{5}{|c|}{$\begin{array}{l}\mathrm{N}^{\circ} \text { observações }-20.767 \\
-2 \text { log verossimilhança }-9.022,23 \\
\text { Qui-quadrado do modelo }-1.430,07 \text { (p-valor }<0,001 \text { ) } \\
\text { Classificados corretamente }-90,5 \% \\
\text { McFadden Pseudo } \mathrm{R}^{2}-0,137 \\
\mathrm{Nota}^{\mathrm{a}} \text { : Coeficientes referentes ao código “0”, não contrata. } \\
\text { Fonte: Paep/Seade } 2001 \text {, PNAD/IBGE } 1992 \text { a } 2003 \text { e Rais/MTE 2002. Elaboração } \\
\text { do autor a partir da transformação dos dados obtidos nas fontes. }\end{array}$} \\
\hline
\end{tabular}

A probabilidade de ser inovadora ou inovadora para o mercado é dada pela fórmula abaixo (Menard, 2001, p. 13):

$$
\mathrm{P}(\mathrm{Y}=1)=\frac{e^{(\alpha+\beta 1 X 1+\beta 2 X 2+\ldots .+\beta k X k)}}{1+e^{(\alpha+\beta 1 X 1+\beta 2 X 2+\ldots .+\beta k X k)}}[2]
$$

$\mathrm{O}$ efeito marginal de $\mathrm{Xk}$ sobre $\mathrm{P}$ é obtido através da derivada parcial de $\mathrm{Y}=\mathrm{Ln} \mathrm{P} / 1-\mathrm{P}$ em relação a Xk. O efeito marginal de Xk sobre $\mathrm{P}$ depende do ponto da curva (ou da superfície) que for considerado. Entretanto, como prova DeMaris (1993), ao contrário do que aponta Roncek (1991), a probabilidade marginal nunca representa exatamente a mudança em $\mathrm{P}$ de um aumento de uma unidade em X. Peng et al. (2002) acrescentam que o conceito de probabilidade marginal não é útil 
para explicar modelos de regressão logística. A única maneira de calcular de modo acurado esse montante é computando $\mathrm{P}(\mathrm{x}+1)-\mathrm{P}(\mathrm{x})$ para valores específicos de $\mathrm{x}$. Como em [1] a maior parte das variáveis são dummy, torna-se inviável trabalhar com "casos típicos". Por isso, no presente estudo restringir-nos-emos à análise do sinal e significância das variáveis.

Da tabela acima, é possível observar que o tempo de estudo médio do trabalhador e o tamanho da firma contribuem positivamente com a probabilidade de a firma inovar. A variável de controle SETOR também é estatisticamente significativa, sendo que as empresas de todos os setores contemplados na tabela têm maior propensão a inovar que o setor de referência, as firmas de "outros serviços".

Com relação às variáveis centrais do estudo, as Kibs para as quais foi possível rejeitar as hipóteses nulas auxiliares, ao nível de significância de 0,10, foram: jurídicas, contábeis, de soluções de internet, de comunicação empresarial, de publicidade e propaganda e de gestão empresarial. Ou seja, é possível rejeitar parcialmente a hipótese nula de que as firmas de serviços que contratam Kibs não são mais inovativas que as congêneres que as contratam.

O cálculo da tolerância (inverso do Variance Inflation Factor - VIF), efetuado por meio de regressão de Mínimos Quadrados Ordinários - conforme sugerido por Menard (2002) - indica que existe baixo nível de colinearidade entre as variáveis do modelo, tendo em vista que os valores recomendados são muito superiores ao limite mínimo recomendado de 0,20 , conforme pode ser observado na tabela abaixo:

Tabela 5

Tolerância das variáveis dos modelos

\begin{tabular}{l|c||c|c}
\hline Variáveis & Coeficiente & Variáveis & Coeficiente \\
\hline JUR & 0,925 & COM & 0,784 \\
\hline CONT & 0,831 & TMKT & 0,881 \\
\hline SOFT & 0,667 & PROP & 0,822 \\
\hline DADO & 0,875 & GEST & 0,900 \\
\hline SITE & 0,560 & TEST & 0,907 \\
\hline NET & 0,539 & Ln $(P O)$ & 0,848 \\
\hline COMP & 0,739 & SETOR & 0,879 \\
\hline
\end{tabular}

Fonte: Paep/Seade (2001), PNAD/IBGE (1992 a 2003) e Rais/MTE (2002).

Elaboração do autor a partir da transformação dos dados obtidos nas fontes.

\section{Conclusões}

As abordagens mais modernas de políticas de inovação, desenvolvidas no âmbito da União Europeia (Lengrand et al., 2002), posteriormente acatadas pela 
Luis Claudio Kubota

OECD (OECD, 2005c), enfatizam as chamadas políticas de terceira geração, e revelam um papel importante para as Kibs e a inovação de serviços.

No âmago da abordagem europeia está o conceito de Economia Baseada em Conhecimento (EBC). A EBC apresenta três características com consideráveis impactos na natureza da inovação: i) a emergência dos serviços e intangíveis; ii) o rápido desenvolvimento das tecnologias de informação e comunicação e a Sociedade da Informação; e iii) os novos papéis do conhecimento, aprendizado organizacional e recursos humanos.

Para Lengrand et al. (2002), a inovação em um EBC não é baseada apenas em pesquisa e desenvolvimento e tecnologia, mas também em habilidades gerenciais e de mercadologia, e conhecimento organizacional, social, econômico e administrativo. Como os tipos e fontes de conhecimentos requeridos para inovações de maior porte tornam-se mais diversos, há maior ênfase na colaboração e uma tendência às inovações serem produzidas por uma rede de atores, em vez de serem desenvolvidas por indivíduos ou organizações autônomas. Conforme pode ser observado dessa discussão europeia e da OECD, a natureza mais incremental e menos tecnológica da inovação de serviços, e a importância da interação - e, por conseguinte, das Kibs - para a inovação, estão perfeitamente alinhadas com os mais recentes desenvolvimentos de políticas para inovação nos países desenvolvidos.

O presente estudo procurou investigar empiricamente se a visão de que as Kibs contribuem para a inovação tecnológica de produto e/ou processo de seus clientes - nesse caso, do próprio setor de serviços - encontra respaldo na Pesquisa da Atividade Econômica Paulista (Paep) 2001. Os resultados indicam que os serviços para os quais essa afirmativa se confirma foram: jurídicos, contábeis, de soluções de internet, de comunicação empresarial, de publicidade e propaganda e de gestão empresarial. Adotando-se uma visão mais restrita da inovação tecnológica - é preciso qualificar a noção defendida na literatura de que as Kibs contribuem para a inovação tecnológica de produto e/ou processo de seus clientes. É preciso analisar caso a caso. É provável que estudo semelhante em pesquisa que contemple outros tipos de inovação, tais como a organizacional e a de mercado, apresente resultados mais positivos para as Kibs. Infelizmente, tal pesquisa ainda não está disponível no Brasil.

Os serviços citados no parágrafo anterior têm um caráter de assessoria/consultoria às empresas clientes. Já os serviços para os quais não foram encontradas evidências de que colaborem no processo inovativo de seus clientes são - com exceção de serviços de desenvolvimento de software - mais operacionais: processamento de dados, gerenciamento de sites, manutenção de computadores e telemarketing. Ou seja, aparentemente o uso de classificações 
industriais para determinar o que são ou não Kibs é problemático, visto que não capturam a natureza da relação entre fornecedor e cliente. Conforme citado anteriormente, para que essa relação represente benefícios do ponto de vista do processo inovativo, é necessária uma intensa colaboração e interação entre as partes.

No que diz respeito a políticas públicas, é necessário investir na ampliação da disponibilidade de dados sobre serviços, e mais especificamente sobre inovação em serviços. As pesquisas sobre inovação em serviços no país ainda restringem-se aos aspectos tecnológicos, quando a literatura apresentada e a mais recente versão do Manual de Oslo ressaltam a importância de inovações organizacionais e de mercado, aspectos já abrangidos em pesquisas de países da OECD. Além disso, a recente edição da Pintec restringe-se - no tocante ao setor de serviços - apenas a telecomunicações, informática e pesquisa e desenvolvimento, escopo incapaz de cobrir as definições mais aceitas sobre as Kibs, que, como demonstrou o presente artigo, podem colaborar para o processo inovativo de seus clientes.

\section{Referências bibliográficas}

ANTONELLI, C. Localized technological change, new information technology and the knowledge and the knowledge-based economy: the European evidence. Journal of Evolutionary Economics, v. 8, p. 177-198, 1998.

ARK, van B.; BROERSMA, L.; HERTOG den P. Services innovation, performance and policy: a review. Hague: Ministry of Economic Affairs, 2003.

BARRAS, R. Towards a theory of innovation in services. Research Policy, v. 15, p. 161173, 1986.

BILDERBEEK, R. et al. Services in innovation: knowledge intensive business services (Kibs) as co-producers of innovation. 1998. (SI4S Synthesis Paper, S3).

CAINELLI, G.; EVANGELISTA, R.; SAVONA, M. The impact of innovation on economic performance in services. The Service Industries Journal, v. 24, n. 1, p. 116-130, Jan. 2004.

DJELLAL, F.; GALLOUJ, F. Innovation surveys for service industries: a review. In: CONFERENCE Innovation and Enterprise Creation: Statistics and Indicators, France, 2000.

DREJER, I. Identifying innovation in surveys of services: a Schumpeterian perspective. Research Policy, v. 33, p. 551-562, 2004.

FREIRE, C. E. T. Um estudo sobre os serviços intensivos em conhecimento no Brasil. In: DE NEGRI, J. A.; KUBOTA, L. C. (Org). Estrutura e dinâmica do setor de serviços no Brasil. Brasília: Ipea, 2006.

GALLOUJ, F. Innovation in services and the attendant old and new myths. Journal of Socio-Economics, v. 31, p. 137-154, 2002. 
Luis Claudio Kubota

GALLOUJ, F.; WEINSTEIN, O. Innovation in services. Research Policy, v. 26, p. 537$556,1997$.

HERTOG den P. Knowledge-intensive business services as co-producers of innovation. International Journal of Innovation Management, v. 4, n. 4, p. 491-528, 2000.

; BROERSMA, L.; ARK van B. On the soft side of innovation: services innovation and its policy implications. De Economist, v. 151, n. 4, p. 433-452, 2003.

HIPP, C. et al. The incidence and effects of innovation in services: evidence from Germany. International Journal of Innovation Management, v. 4, n. 4, p. 471-453, 2000.

IBGE. PESQUISA anual de serviços 2002. Rio de Janeiro: IBGE, 2004. 62p.

KATSOULACOS, Y.; TSOUNIS, N. Knowledge-intensive business services and productivity growth: the Greek evidence. In: BODEN, M.; MILES, I. (Ed.). Services and the knowledge-based economy. London: Continuum, 2000. 286p.

KOX, H. L. M. Growth challenges for the Dutch business services industries: international comparison and policy issues. Hague: CPB Netherlands Bureau for Economic Policy Analysis, 2002.

LENGRAND, L. et al. Innovation tomorrow, innovation policy and the regulatory framework: making innovation an integral part of the broader structural agenda. Luxemburgo: Directorate-General for Enterprise, European Commission, 2002.

LÖÖF, H. Outsourcing, innovation and firm performance in service and manufacturing industries. In: CONFERENCE: Innovation and Enterprise Creation: Statistics and Indicators. França, 2000. Disponível em: <http://www2.arnes.si/ korpl2/FM/FMredni/seminar/teme-inov.model/Innov+\%20Firm\%20Performance.doc $>$. Acesso em: 11 mar. 2006.

LOPES, L. F.; DODINHO, M. M. Services innovation and economic performance: an analysis at the firm level. Danish Research Unit for Industrial Dynamics, 2005. (DRUID Working Paper, n. 05-08).

MENARD, S. Applied logistic regression analysis. Thousand Oaks: Sage Publications, 2002.

MILES, I. Services innovation: a reconfiguration of innovation studies. Manchester: The University of Manchester, 2001. (Discussion Paper Series).

Services innovation: coming of age in the knowledge-based economy. International Journal of Innovation Management, v. 4, n. 4, 371-389, 2000.

NÄHLINDER, J. Innovation in knowledge intensive business services: state of the art and conceptualizations. Linköping: Linköping University, 2002. (Working paper).

OECD. Promoting innovation in services. Paris: OECD. DSTI/STP/TIP(2004)4/FINAL. 14 Oct. 2005a.

Oslo manual: guidelines for collecting and interpreting innovation data. $3^{\text {rd }}$ ed. Paris: OECD, 2005b.

Governance of innovation systems. Paris: OECD, 2005c. v. 1,361p. 
PENG, C.Y. J. et. al. The use and interpretation of logistic regression in higher education journals: 1988-1999. Research in Higher Education, v. 43, n. 3, Jun. 2002.

RONCEK, D. W. Using logit coefficients to obtain the effects of independent variables on changes in probabilities. Social Forces, v. 70, n. 2, p. 509-518, 1991.

SOETE, L.; MIOZZO, M. Internationalization of services: a technological perspective. Technological Forecasting and Social Change, v. 67, n. 2-3, p. 159-185, 2001.

SUNDBO, J.; GALLOUJ, F. Innovation in services. 1998. (SI4S Synthesis Paper S2).

TETHER, B. Do services innovate (differently)? Manchester: The University of Manchester, 2004. (CRIC Discussion Paper n. 66).

; MILES, I. Surveying innovation in services: measurement and policy interpretation issues. In: CONFERENCE Innovation and Enterprise Creation: Statistics and Indicators. France, 2000.

et al. Innovation in the service sector: analysis of data collected under the community innovation survey (CIS-2). Manchester: The University of Manchester \& UMIST, 2002. (CRIC Working Paper, n. 11).

TOMLINSON, M. Information and technology flows from the service sector: a UK-Japan comparison. In: ANDERSEN, B. et. al. (Ed.). Knowledge and innovations in the new service economy. Cheltenham: E. Elgar, 2000. 314p.

UCHUPALANAN, K. Competition and IT-based innovation in banking services. International Journal of Innovation Management, v. 4, n. 4, p. 455-489, 2000. 